\title{
Net-particle number fluctuations in a hydrodynamic de- scription of heavy-ion collisions
}

\author{
Volodymyr Vovchenko ${ }^{1, *}$, Volker Koch ${ }^{1}$, and Chun Shen $^{2,3}$ \\ ${ }^{1}$ Nuclear Science Division, Lawrence Berkeley National Laboratory, Berkeley, CA 94720, USA \\ ${ }^{2}$ Department of Physics and Astronomy, Wayne State University, Detroit, Michigan 48201, USA \\ ${ }^{3}$ RIKEN BNL Research Center, Brookhaven National Laboratory, Upton, NY 11973, USA
}

\begin{abstract}
Utilizing viscous hydrodynamic simulations of heavy-ion collisions, we study the behavior of cumulants of (net-)(anti)proton number distributions at RHIC beam energy scan energies, incorporating non-critical contributions like baryon conservation and excluded volume. The experimental data on net-proton cumulants at $\sqrt{s_{\mathrm{NN}}}>20 \mathrm{GeV}$ are consistent with simultaneous effects of global baryon conservation and repulsive interactions in baryon sector, whereas the data at lower collision energies show possible indications for sizable attractive interactions among baryons. We discuss the behavior of factorial cumulants in addition to the ordinary cumulants, and also address the quantitative difference between proton and baryon number cumulants.
\end{abstract}

\section{Introduction}

As a sensitive probe of the QCD phase structure [1,2], fluctuations of (net-)proton number are among the most valuable observables in the beam energy scan program at RHIC [3], as well as in other heavy-ion experiments [4-6]. The recent experimental data of the STAR Collaboration $[7,8]$ indicate a possible non-monotonic energy dependence of the net-proton kurtosis, which, if confirmed, may indicate presence of the QCD critical point [9].

A quantitative theoretical description of fluctuations is challenging. The bulk observables in heavy-ion collision are commonly well described by relativistic hydrodynamics [1012]. Event-by-event fluctuations, however, are affected by several additional mechanisms like baryon number conservation $[13,14]$, the smearing of fluctuations due to momentum cuts [15], volume fluctuations [16, 17], and other effects. The two main issues mentioned above have recently been addressed at the LHC energies in Refs. [18], utilizing the approximate boost invariance of heavy-ion collisions at those energies. Here these considerations are extended to RHIC-BES energies.

\section{Method}

The details of our simulations setup can be found in Ref. [19]. We study 0-5\% Au-Au collisions at various collision energies from the RHIC-BES program utilizing viscous hydrodynamics simulations within MUSIC [20] from Ref. [21]. The particlization of the QCD

\footnotetext{
*e-mail: vovchenko@lbl.gov
} 

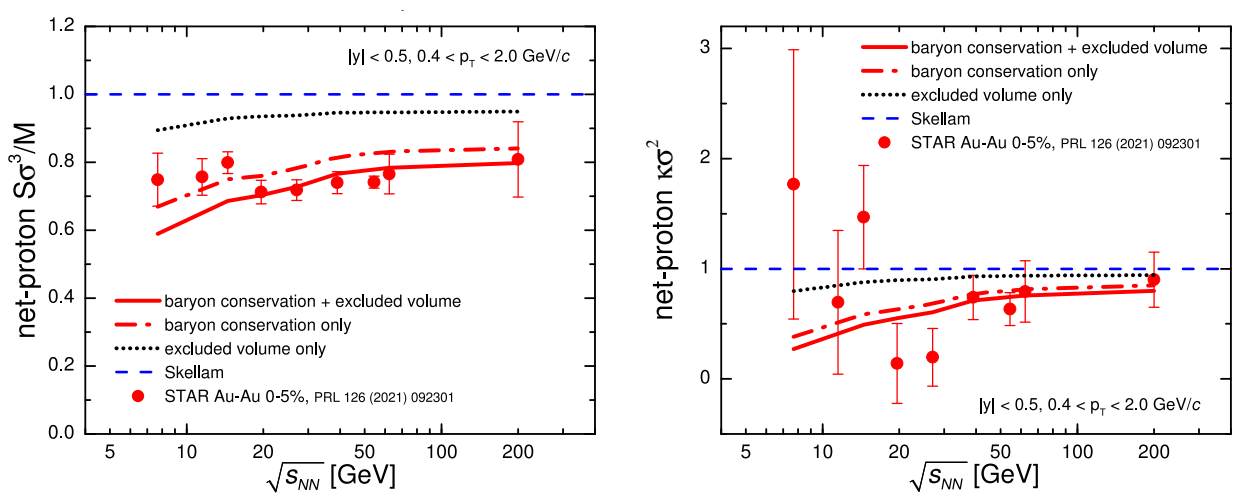

Figure 1. Collision energy dependence of the net-proton cumulant ratios $\kappa_{3} / \kappa_{1} \equiv S \sigma^{3} / M$ (left) and $\kappa_{4} / \kappa_{2} \equiv \kappa \sigma^{2}$ (right) in $0-5 \%$ Au-Au collisions at RHIC BES energies in a non-critical scenario compared with the STAR measurements $[19,24]$.

fluid takes place at a constant "switching" energy density of $\varepsilon_{\mathrm{sw}}=0.26 \mathrm{GeV} / \mathrm{fm}^{3}$ [21]. The cumulants of proton and baryon number are calculated at particlization analytically via a generalized Cooper-Frye procedure which incorporates effects of the excluded volume and baryon conservation. This is performed in two steps:

1. The proton and baryon number cumulants within the experimental acceptance are calculated in the grand-canonical limit, i.e. neglecting the exact global conservation of baryon number.

2. The calculated cumulants are corrected for baryon conservation.

In the first step the emission of particles from each hypersurface can be calculated independently, given the grand-canonical limit of these calculations. The joint baryon-proton cumulants $\kappa_{n, m}^{B^{ \pm}, p^{ \pm}, \text {gce }}\left(\Delta p_{\text {acc }}\right.$ ) of accepted particles correspond to the sum of contributions from each element, where each term is calculated by (i) calculating the distribution of emitted (anti)baryons using the grand-canonical susceptibilities of the excluded volume HRG model [22], then (ii) folding with the binomial distribution to model the momentum acceptance, and (iii) folding with another binomial distribution to separate protons from all baryons. The cumulants $\kappa_{n, m}^{B^{ \pm}, p^{ \pm}, \text {gce }}\left(x_{i} ; \overline{\Delta p_{\text {acc }}}\right)$ of particles outside the acceptance are calculated in the same way.

In the second step, a correction for baryon number conservation is performed. For this purpose we utilize a generalized subensemble acceptance method (SAM) of Ref. [23]. The SAM expresses the joint (net-)(anti-)proton number/net-baryon number cumulants inside the acceptance subject to global baryon conservation in terms of the grand-canonical joint cumulants both inside and outside the acceptance, i.e. it provides the mapping

$$
\kappa_{n, m}^{B, p, \mathrm{ce}}\left(\Delta p_{\text {acc }}\right)=\tilde{\mathcal{S}}\left[\kappa_{n, m}^{B, p, \text { gce }}\left(\Delta p_{\text {acc }}\right), \kappa_{n, m}^{B, p, \text { gce }}\left(\overline{\Delta p_{\text {acc }}}\right)\right] .
$$

\section{Results}

\subsection{Net proton cumulant ratios}

Figure 1 shows the collision energy dependence of the net proton cumulant ratios $\kappa_{3} / \kappa_{1} \equiv$ $S \sigma^{3} / M$ and $\kappa_{4} / \kappa_{2} \equiv \kappa \sigma^{2}$ in comparison with the experimental data of the STAR Collaboration [24]. Both the baryon conservation and excluded volume suppress the two ratios relative 
to the baseline of unity, and lead to a monotonic collision energy dependence. It is clear that baryon conservation has a larger influence at all energies compared to the excluded volume, however, both effects are necessary to obtain a quantitative description of the $S \sigma^{3} / M$ data at $\sqrt{s_{\mathrm{NN}}} \geq 20 \mathrm{GeV}$. At lower energies the data indicate a smaller suppression of $S \sigma^{3} / M$ than predicted by the calculation. As for $\kappa \sigma^{2}$, the STAR data show possible indications for a nonmonotonic collision energy dependence which is not observed in the baseline calculation, however, more precise data at the lowest energies are required to make a robust conclusion.

\subsection{Factorial cumulants}

In addition to the ordinary cumulants, we also explore the (anti)proton correlation functions (factorial cumulants) $\hat{C}_{k}$, which probe genuine multi-particle correlations and thus should be sensitive probes of the critical behavior [15]. We find that the high-order factorial cumulants, $\hat{C}_{3} / \hat{C}_{1}$ and $\hat{C}_{4} / \hat{C}_{1}$, indicate the presence of only mild multi-particle correlations among protons in the non-critical scenario. The second scaled factorial cumulant of protons, $\hat{C}_{2} / \hat{C}_{1}$, shown in Fig. 2, indicates sizable negative two-particle correlations. The calculation results agree with the experimental data at $\sqrt{s_{\mathrm{NN}}} \geq 20 \mathrm{GeV}$ but overestimate the strength of negative correlations at lower collision energies. This may either indicate sizable volume fluctuations at those energies, or presence of attractive interactions among protons.

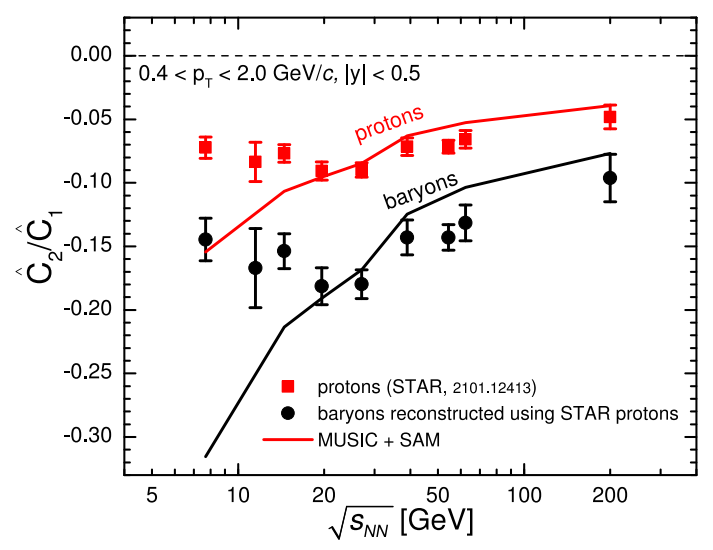

Figure 2. Collision energy dependence of the second scaled factorial cumulant $\hat{C}_{2} / \hat{C}_{1}$ of protons (red line) and baryons (black line) in 0-5\% central Au-Au collisions. The red squares depict the experimental data of the STAR Collaboration for protons [8] while the black circles correspond to baryons reconstructed from the proton data using the binomial distribution.

\subsection{Protons versus baryons}

Our setup allows one to explore the quantitative differences between proton and baryon number fluctuations. It has been argued that baryon cumulants can be reconstructed from the measured proton ones via a binomial unfolding [25], which essentially corresponds to an additional efficiency correction. In particular, the second scaled factorial cumulant of baryons reads $\hat{C}_{2}^{B} / \hat{C}_{1}^{B} \approx 2 \hat{C}_{2}^{p} / \hat{C}_{1}^{p}$, the corresponding experimental data of the STAR Collaboration based on this relation is shown in Fig. 2 by black symbols. The model calculations of two-particle baryon correlations agree well with the reconstructed STAR data for baryons 
at $\sqrt{s_{\mathrm{NN}}} \geq 20 \mathrm{GeV}$, underlying the importance of the unfolding procedure when performing comparisons to theoretical calculations of baryon fluctuations.

\section{Summary}

We analyzed proton number fluctuations within the hydrodynamic description of heavy-ion collisions in a non-critical scenario. The experimental data of the STAR Collaboration at $\sqrt{s_{\mathrm{NN}}}>20 \mathrm{GeV}$ are consistent with simultaneous effects of global baryon conservation and repulsive interactions in baryon sector while the data at lower collision energies show possible indications for sizable attractive interactions among baryons. Our calculations also reveal essential quantitative differences between proton and baryon number cumulants.

\section{References}

[1] M.A. Stephanov, K. Rajagopal, E.V. Shuryak, Phys. Rev. Lett. 81, 4816 (1998), hep-ph/9806219

[2] M.A. Stephanov, K. Rajagopal, E.V. Shuryak, Phys. Rev. D 60, 114028 (1999), hep-ph/9903292

[3] A. Bzdak, S. Esumi, V. Koch, J. Liao, M. Stephanov, N. Xu, Phys. Rept. 853, 1 (2020), 1906.00936

[4] M. Gazdzicki, P. Seyboth, Acta Phys. Polon. B 47, 1201 (2016), 1506.08141

[5] S. Acharya et al. (ALICE), Phys. Lett. B 807, 135564 (2020), 1910. 14396

[6] J. Adamczewski-Musch et al. (HADES), Phys. Rev. C 102, 024914 (2020), 2002.08701

[7] J. Adam et al. (STAR), Phys. Rev. Lett. 126, 092301 (2021), 2001.02852

[8] M. Abdallah et al. (STAR) (2021), 2101.12413

[9] M.A. Stephanov, Phys. Rev. Lett. 107, 052301 (2011), 1104. 1627

[10] C. Gale, S. Jeon, B. Schenke, Int. J. Mod. Phys. A 28, 1340011 (2013), 1301.5893

[11] U. Heinz, R. Snellings, Ann. Rev. Nucl. Part. Sci. 63, 123 (2013), 1301. 2826

[12] C. Shen, L. Yan, Nucl. Sci. Tech. 31, 122 (2020), 2010. 12377

[13] A. Bzdak, V. Koch, V. Skokov, Phys. Rev. C 87, 014901 (2013), 1203.4529

[14] V. Vovchenko, O. Savchuk, R.V. Poberezhnyuk, M.I. Gorenstein, V. Koch, Phys. Lett. B 811, 135868 (2020), 2003. 13905

[15] B. Ling, M.A. Stephanov, Phys. Rev. C 93, 034915 (2016), 1512.09125

[16] M.I. Gorenstein, M. Gazdzicki, Phys. Rev. C 84, 014904 (2011), 1101.4865

[17] V. Skokov, B. Friman, K. Redlich, Phys. Rev. C 88, 034911 (2013), 1205. 4756

[18] V. Vovchenko, V. Koch, Phys. Rev. C 103, 044903 (2021), 2012.09954

[19] V. Vovchenko, V. Koch, C. Shen (2021), 2107.00163

[20] B. Schenke, S. Jeon, C. Gale, Phys. Rev. C 82, 014903 (2010), 1004 . 1408

[21] C. Shen, S. Alzhrani, Phys. Rev. C 102, 014909 (2020), 2003.05852

[22] V. Vovchenko, A. Pasztor, Z. Fodor, S.D. Katz, H. Stoecker, Phys. Lett. B 775, 71 (2017), 1708.02852

[23] V. Vovchenko (2021), 2106.13775

[24] J. Adam et al. (STAR), Phys. Rev. Lett. 126, 092301 (2021), 2001.02852

[25] M. Kitazawa, M. Asakawa, Phys. Rev. C 86, 024904 (2012), [Erratum: Phys.Rev.C 86, 069902 (2012)], 1205.3292 